\title{
De la migration circulaire à l'abandon du territoire local dans le Zarmaganda (Niger)
}

\section{Harouna Mounkaïla}

\section{(2) OpenEdition \\ 1 Journals}

Édition électronique

URL : https://journals.openedition.org/remi/1662

DOI : $10.4000 /$ remi. 1662

ISSN : $1777-5418$

Éditeur

Université de Poitiers

\section{Édition imprimée}

Date de publication : 1 juillet 2002

Pagination : 161-187

ISBN : 2-911627-30-X

ISSN : 0765-0752

\section{Référence électronique}

Harouna Mounkaïla, « De la migration circulaire à l'abandon du territoire local dans le Zarmaganda (Niger) ", Revue européenne des migrations internationales [En ligne], vol. $18-n^{\circ} 2$ | 2002, mis en ligne le 09 juin 2006, consulté le 16 avril 2022. URL : http://journals.openedition.org/remi/1662 ; DOI : https:// doi.org/10.4000/remi.1662

Ce document a été généré automatiquement le 16 avril 2022.

(c) Université de Poitiers 


\title{
De la migration circulaire à l'abandon du territoire local dans le Zarmaganda (Niger)
}

\author{
Harouna Mounkaïla
}

1 Le Zarmaganda ou département de Ouallam (carte $n^{\circ} 1$ ) se situe entre les latitudes $14^{\circ}$ et $16^{\circ}$ Nord et les longitudes $1^{\circ} 62$ et $2^{\circ} 66$ Est. Il couvre une superficie de $22132 \mathrm{~km}^{2}$. Il présente dans l'ensemble un relief très peu contrasté avec une altitude variant de 200 $\mathrm{m}$ au Sud à $350 \mathrm{~m}$ au Nord. Ses formes caractéristiques sont les vallées et les plateaux gréseux portant une brousse tigrée s'étendant du Sud jusqu'au $15^{\mathrm{e}}$ parallèle au Nord. Au-delà de cette latitude, jusqu'à la frontière du Mali se développent des cordons de dunes mortes d'orientation est-ouest. "Berceau des Zarma» comme l'indique son étymologie par opposition aux aires d'extension de ce peuple, cette région a été le support spatial des premières installations des groupes zarma au $\mathrm{xvI}^{\mathrm{e}}$ siècle. Elle est aujourd'hui l'une des régions les plus déshéritées du Niger en raison non seulement de son enclavement et de sa pauvreté, mais aussi de son extrême vulnérabilité économique et de l'insécurité alimentaire quasi permanente qui y sévit depuis 30 ans. Cette insécurité alimentaire peut prendre parfois des allures de crise pendant les années de paroxysmes climatiques comme ce fut le cas en 1973, 1984 et 1987. Les aléas climatiques semblent avoir joué et jouent encore un rôle prépondérant dans l'insécurité alimentaire qui prévaut aujourd'hui dans la région.

2 Loin de s'attacher exclusivement à une agriculture dont les résultats ne répondent pas chaque année à leur espérance, les populations zarma du Zarmaganda, ont déployé leur ingéniosité dans la recherche quotidienne d'autres sources de revenus. Parmi les réactions diverses à l'insécurité alimentaire permanente la plus fréquemment observée demeure la mobilité spatiale. Selon l'ampleur des déficits vivriers, cette mobilité peut prendre plusieurs formes allant de la circulation migratoire, pris au sens d'un va-etvient entre pays de départ et pays d'arrivée, à l'abandon du territoire local.

3 La préoccupation d'ensemble de cet article consiste à mettre en évidence les interactions complexes entre insécurité alimentaire et migration. Il s'agit de 
comprendre comment la seconde s'intègre dans les stratégies paysannes de gestion de la première. Il s'agit également de définir les pratiques et les configurations migratoires qui en résultent. À partir de cette réflexion d'ensemble, on peut aussi apprécier de quelles manières les migrations nées de l'insécurité alimentaire s'inscrivent dans l'espace.

4 Le domaine géographique de l'étude couvre certes le Zarmaganda, mais notre réflexion sera essentiellement bâtie autour des résultats d'une enquête effectuée en 1996 dans deux villages du canton de Tondikiwindi à savoir Taroum et Mogonana, situés respectivement à $158 \mathrm{~km}$ et $162 \mathrm{~km}$ au nord de Niamey (carte ${ }^{\circ} 1$ ).

Carte 1 : Localisation de la zone d'étude

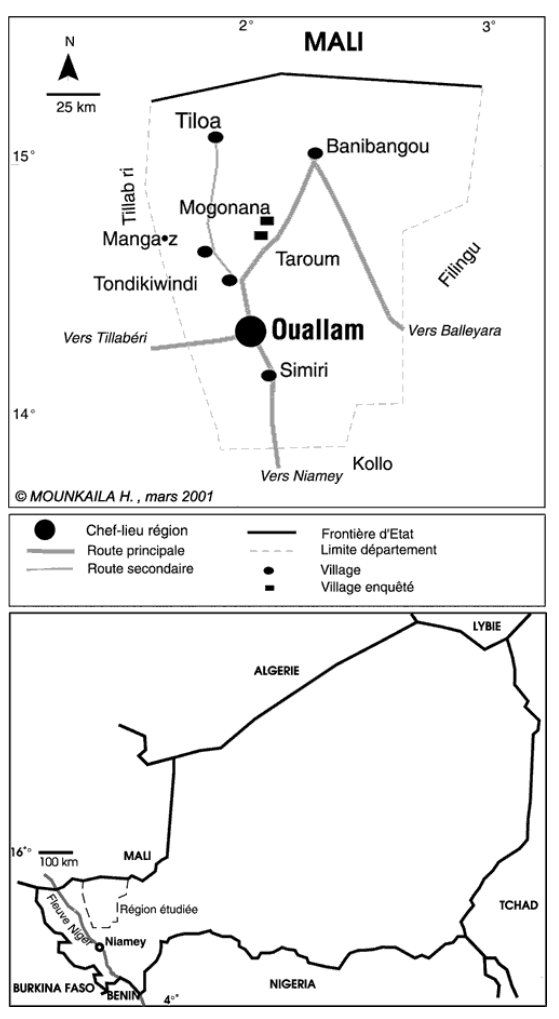

5 La mobilité spatiale est l'une des caractéristiques essentielles des populations sahéliennes qui se sont toujours déplacées pour des raisons diverses. L'espace sahélien est un espace ouvert qui favorise les grands déplacements (Mauny, 1961:220). Cette caractéristique géographique fondamentale constitue l'un des éléments clés de l'interprétation des rapports des hommes à l'espace. Les systèmes de production pastorale et agricole développés par les populations sont fondés sur l'intense mobilité des hommes. Ils se traduisent par certaines façons d'occuper et d'approprier l'espace qui accordent une place centrale à la mobilité spatiale. Les contraintes physiques, parfois politiques et économiques du milieu ont également joué un rôle important dans les rapports entre les groupes sociaux et leur environnement et déterminent aujourd'hui encore l'accélération et la diversité des mouvements de population.

6 Les populations du Zarmaganda ont une tradition séculaire de mobilité géographique en phase avec l'évolution d'un milieu dont les contraintes géographiques sont importantes. Les migrations ont joué un rôle déterminant dans le peuplement de la région. Pendant la période pré-coloniale, il s'agissait essentiellement de migrations de 
peuples (Zachariah, $1978: 4$ ), c'est-à-dire de groupes sociaux entiers. C'est ainsi que, les Zarma, qui seraient originaires du Dirma au Mali, auraient quitté cette région au XVI ${ }^{\mathrm{e}}$ siècle par vagues migratoires pour s'installer dans le Zarmaganda (Urvoy, 1936 : 55 ; Hama, 1967 : 105 ; Sidikou, 1974 : 48-49; Gado, 1980 : 160-161). À partir de ce berceau originel, ils se sont dispersé vers le Sud et le Sud-Est, élargissant ainsi leur distribution spatiale sous la pression de leur croissance démographique, des contraintes du milieu - le Zarmaganda est un milieu ingrat - et de leur capacité à exploiter les ressources de leur environnement. Ces mouvements allaient asseoir définitivement le peuplement effectif de la région qui ne connaîtra pas de réajustements significatifs jusqu'à la colonisation, même si par ailleurs le danger touareg, les querelles internes et les activités de mercenariat auxquelles s'étaient livrées les Wangari ${ }^{1}$ pendant toute la deuxième moitié du $\mathrm{XIX}^{\mathrm{e}}$ siècle avaient créé une situation d'insécurité quasi générale dans la région (Idrissa, $1981: 14$ ).

7 La colonisation va jouer à son tour un tournant décisif dans l'évolution de la pratique migratoire de ces populations. Elle déclencha d'importants mouvements d'éclatement des villages et de dispersion de l'habitat en raison notamment des contraintes de toutes sortes qu'elle imposa aux populations. Ces déplacements qui avaient pour but d'échapper aux méthodes coercitives de domination (Olivier de Sardan, $1984: 174)$ et qui furent favorisés par les famines, la croissance démographique et l'épuisement des terres (Sidikou, $1974: 65)$, ont constitué pendant cette période les seuls remaniements à l'échelle locale des structures d'occupation de l'espace. Cependant, ils ne rendent que partiellement compte de la dynamique globale des migrations impulsées sous la colonisation. En effet, il faut élargir l'analyse aux migrations vers les colonies de la côte atlantique. Ces migrations au départ essentiellement dirigées vers la Gold Coast, étaient motivées par des raisons politiques liées en partie à la rigueur du système politique colonial français. Par la suite, leur champ d'explication s'est étendu aux raisons économiques qui finiront par être les plus déterminantes après que la plupart des contraintes coloniales auront été supprimées. Ces mouvements de population se déclinent essentiellement en migrations de travail rendues indispensables par le nouveau système d'exploitation économique mis en place et fondé sur une division du travail et une logique de complémentarité entre colonies. Dans la nouvelle dynamique d'exploitation économique, le colonisateur français attribua aux colonies de l'intérieur le rôle peu enviable de pourvoyeuses de main-d'œuvre des pôles économiques côtiers dans lesquels ont été développées des cultures d'exportation. L'ancienne colonie du Niger était l'un de ces pays réservoirs de main-d'œuvre et les populations zarma l'une des composantes de cette force de travail. Les mouvements migratoires, résultant de ce système d'exploitation économique, constituent aujourd'hui le creuset de la migration internationale de ces populations.

8 Par ailleurs, parmi les bouleversements introduits par la colonisation, figurent la naissance, puis le développement progressif des migrations rurales-urbaines, plus connues et à juste titre, sous le vocable d'exode rural. Celui-ci n'est pas synonyme ici, comme ailleurs - en Europe notamment -, de dépeuplement des campagnes au profit des villes. Il constitue au contraire une des stratégies de maintien de leur peuplement.

Les sécheresses successives de ces trois dernières décennies et l'insécurité alimentaire qui en résulte dans un contexte économique très précaire ont déclenché de nouveaux courants migratoires et intensifié constamment la circulation migratoire. Dans un tel contexte, le potentiel migratoire du Zarmaganda ne peut qu'augmenter et perpétuer 
l'existence et le développement d'une pratique spatiale séculaire dont les formes sont multiples et variées.

10 Cet article s'articule autour de deux parties. Dans la première, nous aborderons l'étude de l'insécurité alimentaire essentiellement perçue ici comme une contrainte qui appelle la mise en œuvre de stratégies d'adaptation. Enfin, dans une deuxième partie, nous verrons comment la gestion de l'insécurité alimentaire s'exprime à travers des pratiques migratoires diverses qui s'inscrivent soit dans une logique de maintien des populations dans leur territoire local, soit dans la finalité de soustraire les acteurs de cette contrainte majeure.

L'insécurité alimentaire : une donnée constante de l'histoire économique du zarmaganda

11 L'histoire économique du Zarmaganda est faite d'épisodes de famines et de disettes dont certains ont eu des conséquences démographiques dramatiques. De plus, elles se sont le plus souvent accompagnées de mouvements de population.

12 Le problème qui préoccupe aujourd'hui le monde rural nigérien en général et celui du Zarmaganda en particulier est bien évidemment l'insécurité alimentaire. Elle est la conséquence de plusieurs facteurs cumulatifs.

Une insécurité alimentaire liée aux contraintes d'une agriculture à la productivité médiocre

13 L'un des facteurs de l'insécurité alimentaire est l'incertitude pluviométrique. Les aléas climatiques ont une incidence sur la production agricole (figure 1).

Figure 1 : Évolution comparée de la production céréalière et de la pluviométrie de 1969 à 1988 à Ouallam

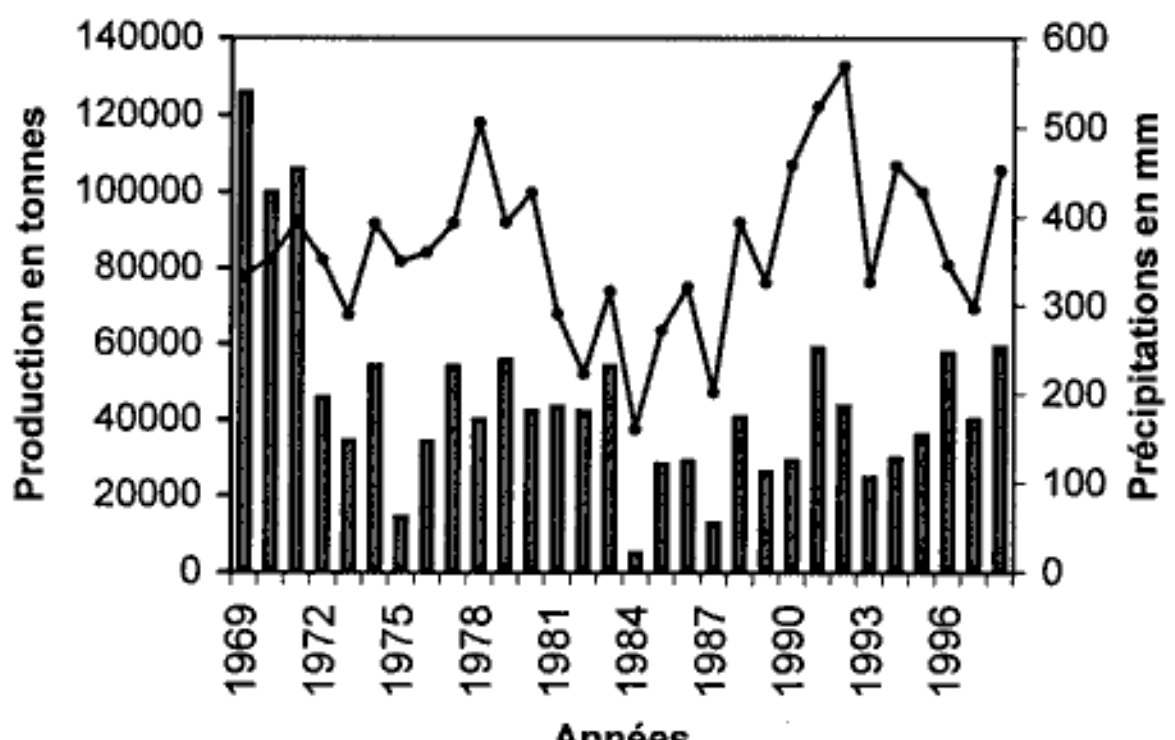

Années

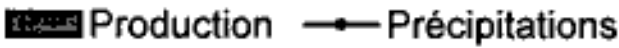

14 La figure met en lumière une instabilité inter-annuelle importante des précipitations et de la production céréalière. On remarque que les années de faible pluviométrie (1973, 1975, 1982, 1984, 1987, 1989 et 1993) sont également des années pendant lesquelles la 
production céréalière a connu une importante baisse. Par exemple, la production de mil de l'année 1984 dans le Zarmaganda représentait seulement $11 \%$ de celle de l'année 1983, c'est-à-dire qu'elle avait baissé de $89 \%$ par rapport à son niveau de 1983 . On observe une évolution fluctuante de la production vivrière en fonction des années et au gré des aléas climatiques et des autres problèmes que connaît l'agriculture vivrière. Par contre, au cours de certaines années de relative bonne pluviométrie comme ce fut le cas en 1978, 1980, 1992, on n'a pas assisté non plus à une augmentation de la production agricole. C'est pourquoi les aléas climatiques ne doivent pas être réduits au seul volume des précipitations. Il faut tenir compte de leur répartition dans le temps. Par ailleurs, le rôle des aléas climatiques dans l'insuffisance de la production doit être relativisé. Si l'irrégularité des précipitations constitue un des facteurs déterminants, elle n'explique pas tout. D'autres facteurs notamment la croissance démographique, l'épuisement des sols et l'inefficacité du système de production constituent des éléments d'explication incontournables. Nous ferons un bref aperçu du contexte géographique et humain dans lequel s'inscrivent les activités agricoles.

Les péjorations de la pluviométrie se sont accompagnées d'un éclaircissement du couvert végétal, voire de sa disparition, exposant les sols dénudés aux vents et aux ruissellements. La disparition du couvert végétal a été accélérée par des actions anthropiques diverses dont la plus importante est la coupe abusive du bois devenue une source de revenus pour de nombreux paysans. Les sols subissent pour cette raison des formes diverses de dégradation. Celle-ci se traduit par la baisse de leur potentiel biologique et de leur fertilité. L'encroûtement pelliculaire sur les glacis, la salinisation dans les bas-fonds, sont autant de facteurs physiques qui accroissent l'érosion mécanique par le renforcement de l'agressivité et de l'efficacité érosives des vents et des ruissellements. Dans l'ensemble de la région, les aptitudes agricoles des sols ne sont jamais très élevées alors que les contraintes peuvent se révéler très importantes. La très forte sensibilité des sols à l'érosion hydrique et éolienne explique aujourd'hui leur niveau de dégradation. Cette sensibilité est devenue d'autant plus grande que l'extension des superficies cultivées à des terres de fertilité marginale est une réponse apportée à la baisse des rendements. Celle-ci est le plus souvent la conséquence de la réduction du temps des jachères (elle est en moyenne de trois ans à Taroum et Mogonana) qui, jusque-là, permettaient par des processus biologiques (accumulation de matières organiques en surface et formation de litière, amélioration de l'infiltration, élimination des parasites de culture etc.) la reconstitution des sols. La réduction de ce système de maintien de la fertilité le plus efficace sur le plan agronomique et le plus économique est la conséquence d'un accroissement de la population qui rend aujourd'hui l'extension de l'agriculture presque impossible puisque la terre est finie. Cette réduction de la durée de la jachère ne s'est pas accompagnée du développement d'autres techniques de fertilisation des sols à l'exception du paillage pratiqué timidement çà et là et de quelques techniques non encore vulgarisées initiées sous la bénédiction des projets de développement financés essentiellement par les bailleurs de fonds extérieurs.

16 L'état du système d'exploitation a contribué aussi à déclencher la crise des milieux. En effet, le mode extensif de l'exploitation de l'espace qui a longtemps été possible à cause de «l'illusion » que l'espace était infini connaît ses limites en raison surtout de la croissance démographique. L'évolution démographique ne s'est pas accompagnée d'une évolution concomitante des techniques culturales et du mode de production. Dans ces conditions, il est important de remarquer que le facteur humain a joué et continue de 
jouer un rôle important dans l'appauvrissement des sols et détient une part de responsabilité dans l'insécurité alimentaire qui prévaut aujourd'hui dans la région.

Une histoire économique faite de crises alimentaires

Avant de procéder à l'inventaire des crises alimentaires survenues dans le Zarmaganda, il convient au préalable de définir la notion de famine. Celle-ci est «une forme de malnutrition générale prolongée et persistante, qui dure plusieurs mois et affecte la majorité d'une population rurale sur une zone plus ou moins étendue qui provoque une totale désorganisation économique et sociale et la mort massive par inanition " (Azoulay et Dillon, 1993: 112). La chronologie qui va suivre s'appuie sur des sources diverses (travaux d'historiens, rapports d'administrateurs coloniaux et tradition orale).

La reconstitution des phénomènes de crises alimentaires dans leur contexte historique est possible dès lors que la mémoire collective conserve leurs repères chronologiques en les associant à certains événements marquants de l'histoire de ces sociétés. Parmi les conséquences des famines une retient particulièrement notre attention. Il s'agit de leurs conséquences démographiques en particulier les mouvements de population. Le tableau $\mathrm{n}^{\circ} 1$ nous permet d'apprécier les dimensions démographiques des crises de subsistance dans le Zarmaganda.

Les aléas de la pluviométrie et les calamités naturelles, notamment les ravages de criquets sédentaires ou pèlerins, ont constitué une menace permanente pour les récoltes qu'ils réduisent parfois à néant. Le plus souvent ces facteurs se cumulent pour entraîner la famine ou la disette. Ainsi en est-il des famines de 1901-1903, 1913-1914, 1954-1955, 1965-1966. Si ces facteurs sont des éléments déclencheurs des crises de subsistance, leur rôle dans l'explication de ces phénomènes doit être relativisé. La destruction des récoltes par des essaims de criquets ne peut à elle seule déclencher une crise de subsistance grave. Il faut que d'autres conditions soient réunies notamment que les greniers soient vides ou que les déficits vivriers se soient succédés sur plusieurs années et que cette situation bénéficie d'un contexte économique caractérisé par la faible disponibilité ou le manque d'un capital sur pied (bétail). Par ailleurs la classification entre les causes et les conséquences des crises alimentaires doit être dépassée, une conséquence d'une première cause pouvant être à l'origine d'une nouvelle crise alimentaire.

Il est difficile de parler de cycle dans la récurrence des famines d'autant plus qu'elles n'interviennent pas à des intervalles réguliers. On constate cependant que depuis ces vingt dernières années, les crises alimentaires surviennent à des périodes de plus en plus rapprochées. En vingt ans, de 1970 à 1990, la région a connu quatre épisodes de famines : 1973-1974, 1982, 1984 et 1987. 
Tableau 1 : Crises alimentaires survenues au $\mathrm{Xx}^{\mathrm{e}}$ siècle dans le Zarmaganda

\begin{tabular}{|c|c|c|c|}
\hline Année & Termes locaux & Causes & Conséquences \\
\hline 1900-1903 & Izénéré (vente d'enfants) & $\begin{array}{l}\text { Sécheresse + } \\
\text { invasion de } \\
\text { criquets }\end{array}$ & $\begin{array}{l}\text { migration massive vers le } \\
\text { Nigeria }\end{array}$ \\
\hline 1913-1914 & $\begin{array}{l}\text { Ganda-béri (grande brassée) } \\
\text { ou Yollomorou (caresser ses } \\
\text { tresses) }\end{array}$ & Sécheresse & $\begin{array}{l}\text { Dispersion, création des } \\
\text { villages de culture, migration } \\
\text { vers Dallol Bosso, Nord } \\
\text { Nigeria et fleuve Niger. }\end{array}$ \\
\hline 1929-1931 & $\begin{array}{l}\text { Soudan : Soudan beri ou } \\
\text { Zama kano (couteau } \\
\text { tranchant) et soudan kainé } \\
\text { (petit soudan) ou Doadjiré } \\
\text { (année du criquet) }\end{array}$ & $\begin{array}{l}\text { Invasion de } \\
\text { criquets }\end{array}$ & $\begin{array}{l}\text { Dispersion, peuplement des } \\
\text { villages de culture, migration } \\
\text { vers le Dallol Bosso, le Nord } \\
\text { Nigeria et le fleuve Niger. }\end{array}$ \\
\hline 1944 & $\begin{array}{l}\text { Yéda Konadjiré (année de } \\
\text { l'approvisionnement à partir } \\
\text { de Yéda) }\end{array}$ & $\begin{array}{l}\text { Insuffisance } \\
\text { des pluies }\end{array}$ & $\begin{array}{l}\text { Pas entrainé des mouvements } \\
\text { de population }\end{array}$ \\
\hline $1954-1955$ & $\begin{array}{l}\text { Garo djiré (année de la } \\
\text { farine de manioc) }\end{array}$ & $\begin{array}{l}\text { Insuffisance } \\
\text { des pluies }\end{array}$ & Faible migration \\
\hline 1965-1966 & Banda-bari (tourner le dos) & $\begin{array}{l}\text { Ravage criquets } \\
\text { sédentaires }\end{array}$ & migration massive à Niamey \\
\hline 1973-1974 & $\begin{array}{l}\text { Hamma djiré (Année du } \\
\text { sorgho) }\end{array}$ & Sécheresse & $\begin{array}{l}\text { migration massive à Niamey, } \\
\text { mouvements de colonisation } \\
\text { vers les arrondissements de } \\
\text { Say et Kollo. }\end{array}$ \\
\hline 1982 & Sapto (départ précipité) & $\begin{array}{l}\text { Insuffisance } \\
\text { des pluies }\end{array}$ & $\begin{array}{l}\text { migration des populations du } \\
\text { canton de Tondikiwindi vers } \\
\text { les arrondissements de Kollo } \\
\text { et Say }\end{array}$ \\
\hline 1984 & $\begin{array}{l}\text { Dazar (éparpillement) ou } \\
\text { Djaba-kourou (traf̂ner son } \\
\text { boubou) ou koga (sécheresse) }\end{array}$ & Sécheresse & $\begin{array}{l}\text { migration massive à Niamey } \\
\text { et dans les arrondissements } \\
\text { de Say et Kollo }\end{array}$ \\
\hline
\end{tabular}

Sources : Gado, B.A (1992) ; Sidikou, A. H et documents des archives nationales du Niger. Informations recueillies au cours des enquêtes.

21 Les disettes et les famines entraînent le plus souvent des déplacements définitifs qui sont orientés dans la plupart des cas vers d'autres régions rurales mais aussi vers la ville de Niamey. Pendant longtemps, la vallée du fleuve Niger, le Dallol Bosso, le Nord Nigeria ont constitué des lieux d'accueil privilégiés pour la majorité de ces groupes. Depuis une vingtaine d'années, les mouvements semblent se diriger vers les départements de Say et Kollo où les migrants zarma se sont constitués en importants îlots de colonisation (carte $\mathrm{n}^{\circ} 2$ ). 
Carte 2 : Évolution spatiale des migrations liées aux famines et aux sécheresses survenues au Xx siècle dans l'Ouest du Niger
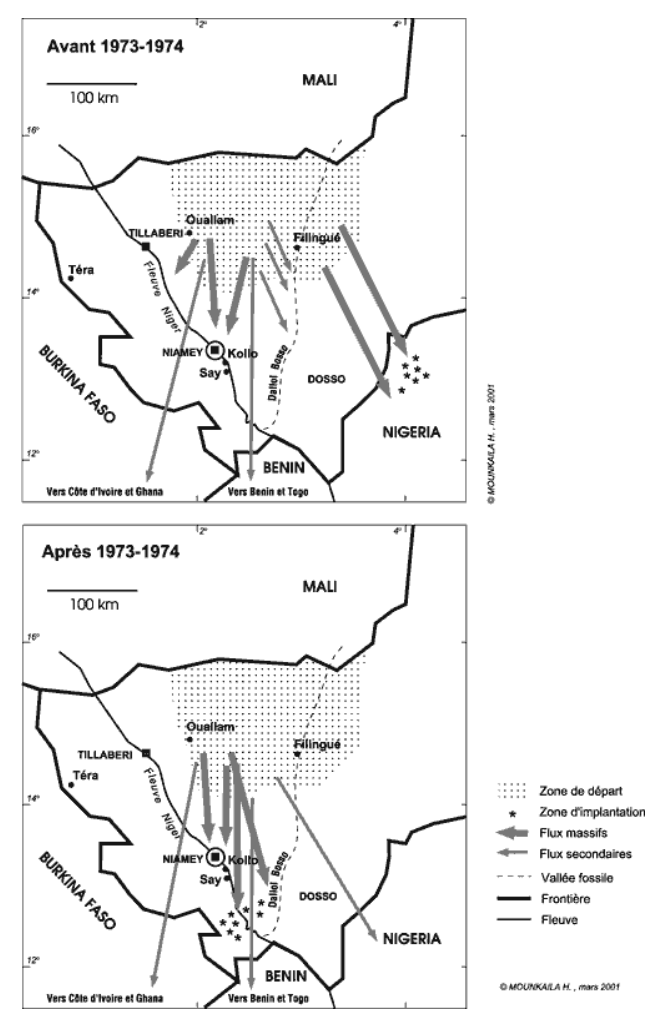

22 Deux raisons principales expliquent cette réorientation migratoire. Il s'agit tout d'abord de la disponibilité en terres de culture, en particulier dans le département de Say. Dans cette région qui présente des conditions agro-climatiques favorables, la présence de maladies endémiques (trypanosomiase, onchocercose) avait longtemps limité la mise en valeur des terres de culture. La deuxième raison tient à la saturation démographique des aires traditionnelles d'accueil notamment la vallée du fleuve Niger et le dallol Bosso.

23 Les disettes et les famines entraînent toujours des déplacements de masse. Ce sont des familles entières qui se déplacent pour échapper à la mort. En 1984 par exemple, toutes les familles de Mogonana à l'exception de quatre, avaient quitté ce village pour s'installer à Niamey. Dans ces circonstances certains villages fournissent à la ville des bouches à nourrir plutôt que des aliments (Ebrahimi-Yaganah, 1996 : 128).

24 Les migrations ont toujours constitué le dernier recours lorsque toutes les solutions locales au problème alimentaire étaient épuisées. Il est difficile cependant de fournir des valeurs chiffrées pour mesurer l'ampleur des déplacements qui sont liés aux famines. À partir de nos enquêtes menées dans deux villages du Nord Zarmaganda, nous avons enregistré le départ définitif de 64 familles sur 146 à Taroum et de 17 familles sur 106 à Mogonana suite à la famine de 1984, soit respectivement $43 \%$ et $16 \%$.

Il est important de souligner le caractère précipité des mouvements de population qui accompagnent les famines comme le montrent les expressions employées pour les nommer. Ainsi, le terme sapto utilisé pour nommer la famine de 1982 dans le canton de Tondikiwindi, exprime cette spontanéité du départ qui ne donne lieu à aucune préparation ; le terme signifiant : départ précipité. Il en est de même de la famine dazaï 
de 1984 qui signifie éparpillement (allusion aux nombreux ressortissants de Ouallam présents dans d'autres départements).

Les crises de subsistance ne sont donc pas nouvelles. Elles ont toujours existé dans l'histoire de ces populations. Cependant, elles n'étaient pas continues dans le temps. Elles intervenaient de façon épisodique et étaient presque toujours suivies de périodes d'abondance. C'est ce qui les différencie des crises de subsistance actuelles qui semblent s'inscrire dans la longue durée.

Les tendances récentes de l'insécurité alimentaire

S'il est un élément qui atteste le mieux la régularité de l'insécurité alimentaire, c'est bien évidemment la soudure. Celle-ci est un type d'insécurité alimentaire qui se caractérise par une déficience temporaire du régime alimentaire. On évoque la soudure lorsque les greniers sont vides alors que s'annonce ou s'installe la saison des pluies. En général, la soudure intervient pendant la période des travaux agricoles au moment où l'agriculteur doit consacrer le maximum de temps à ses cultures. Elle intervient plus tôt ou plus tard en fonction de l'état des récoltes de la saison précédente et peut être de courte durée ou aiguë. Par ailleurs, il peut arriver pour des raisons diverses (paiement de l'impôt, financement de mariages, achat de produits manufacturés, de condiments etc ...), que le paysan vende une partie de sa production qui initialement suffisait tout juste à la consommation annuelle familiale. La vente d'une partie des récoltes, quelle que soit la raison, accélère l'épuisement des réserves et par conséquent précipite aussi la soudure.

Le problème de la soudure se pose donc chaque année compte tenu de l'insuffisance de la production agricole. Lorsque les déficits se succèdent, les paysans n'arrivent plus à constituer des réserves qui pourraient les mettre à l'abri de pénuries alimentaires ultérieures (figure $\mathrm{n}^{\circ} 2$ ). Cette situation accroît leur vulnérabilité et les oblige à adopter des stratégies adaptatives.

Figure 2 :Évolution de la production et des besoins alimentaires de 1985 et 1998 dans le département de Ouallam

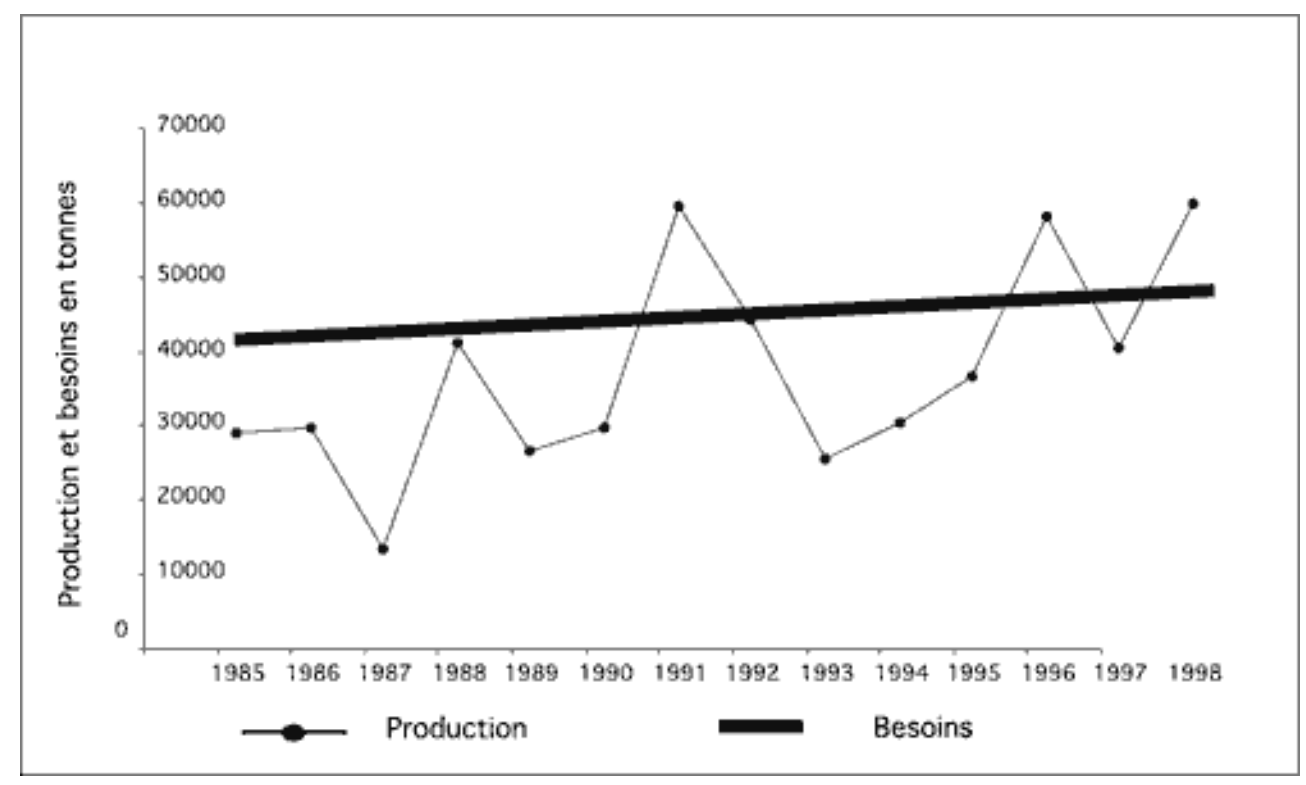

Source : Service d'arrondissement del'agriculture de Ouallam 

pauvreté paysanne, il faut partir ailleurs trouver les ressources monétaires indispensables. Lorsqu'il ne reste dans les greniers que de quoi consommer pendant deux à cinq mois, ou rien du tout, comment ne pas encourager le départ des jeunes surtout lorsqu'on sait que les ressources migratoires sont la seule garantie pour faire face aux besoins alimentaires pendant les cinq à huit autres mois restants de l'année précédant les récoltes suivantes. La migration figure parmi les réponses apportées à l'insécurité alimentaire. Elle est d'ailleurs la principale. Les stratégies d'entraide, de consommation de produits de cueillette et les aides alimentaires ponctuelles ont montré leurs limites. Le recours à l'entraide est rendu aléatoire par le fait que les déficits vivriers concernent généralement de vastes régions et empêchent à coup sûr la solidarité familiale de fonctionner sauf pour certains paysans ayant des parents salariés ou commerçants en ville. Les produits de cueillette (feuilles et fleurs d'arbre, graines sauvages) ne peuvent se substituer au mil, au sorgho ou au maïs dans l'alimentation. La distribution gratuite des vivres est une opération ponctuelle. De plus la quantité de l'aide alimentaire a toujours été largement en deçà des besoins des populations nécessiteuses. Elle ne peut par conséquent les sécuriser. Si aujourd'hui, les famines n'entraînent pas systématiquement comme il y a 67 ans une catastrophe démographique à l'image de celle qui a provoqué en 1931 dans l'ouest du Niger 30000 morts (Dubresson et al., 1994: 79), c'est que les importations alimentaires, les aides alimentaires, la migration et les autres stratégies alternatives permettent d'atténuer leurs effets.

Quand les crises alimentaires surviennent, ce sont des familles entières qui se déplacent. Voici ce que note à ce propos un administrateur colonial dans un rapport de tournée: "Toute la région recensée a été éprouvée par la disette qui a sévi pendant ces dernières années. Beaucoup d'indigènes sont morts et un grand nombre sont partis au Nigeria ou dans les colonies et cercles voisins" (Tornezy, 1934)2. La famine de 1931 avait entraîné l'émigration de 29000 personnes dans les cercles de l'Ouest du Niger (Derriennic, 1977 : 128). Aujourd'hui encore, émigrer constitue la principale stratégie d'adaptation à ces crises.

Les populations ont recours aussi à d'autres activités secondaires notamment la vente d'animaux, de bois, de la paille, des produits artisanaux et les cultures de contre-saison. À Taroum et Mogonana, certains paysans, notamment ceux qui n'ont pas pu partir en migration tentent difficilement de s'adapter à ce contexte en vendant du bois de feu aux camionneurs de Niamey. Dans ces deux villages, les paysans ne pratiquent pas de culture de contre-saison en raison de l'absence d'eau de surface pendant la saison 
sèche. Les cinq puits qui existent ne permettent pas non plus de contourner ce facteur limitant d'autant plus que leurs débits sont faibles. Dans le Zarmaganda en général, les paysans adoptent rarement la pratique des cultures de contre-saison et pourtant celleci figure parmi les stratégies de sécurisation alimentaire des populations préconisées par l'État depuis 1984.

Face à ces crises de subsistance, nous savons combien furent inefficaces les mesures préconisées d'abord par l'administration coloniale, ensuite par l'État post-colonial. L'administration coloniale, en son temps, institua les «greniers de réserve » que les populations ne tardèrent pas à qualifier de "anassara barma» (grenier du blanc) en raison du caractère brutal de leur application et surtout de leur impopularité. Cette initiative, louable dans son principe, fut recommandée en 1933 par un inspecteur de l'administration coloniale au terme d'une enquête menée sur la famine de 1931.

Les pouvoirs post-coloniaux prenant le relais de l'administration coloniale après l'indépendance, n'ont guère été et ne sont pas en mesure d'enrayer le spectre de l'insécurité alimentaire. Les différentes mesures préconisées par les pouvoirs publics pour sécuriser les populations sur le plan alimentaire vont de l'intensification et de la diversification de la production agricole à la distribution gratuite des vivres en passant par la bonne gestion des stocks vivriers et leur commercialisation, l'approvisionnement des banques céréalières et la pratique des cultures de contre-saison.

Lorsqu'on insiste sur l'importance des crises alimentaires dans l'accélération des migrations, on a logiquement tendance à croire qu'un accroissement de la production agricole se traduirait par une réduction significative des départs. Et pourtant, il n'en est rien. D'où la nécessité de relativiser l'importance de l'insuffisance alimentaire dans la dynamique migratoire actuelle. Les migrations se poursuivent même lorsque la production agricole est satisfaisante dans un village. D'autres facteurs agissent simultanément. Ils puisent leur force notamment dans le contexte socio-économique de la région et les dynamiques propres au phénomène migratoire.

La migration comme stratégie de gestion de l'insécurité alimentaire

Dans le Zarmaganda, les pratiques migratoires relatives à la gestion de l'insécurité alimentaire, s'inscrivent essentiellement dans deux types de stratégies. La première, la plus couramment observée, consiste à l'affronter en s'y adaptant à travers la recherche hors du territoire local des ressources complémentaires à celles qu'offre le milieu. La seconde qui n'est pas une stratégie d'adaptation en tant que telle, la plus épisodique, vise principalement à y soustraire les populations les plus vulnérables par l'abandon des villages menacés.

Stratégie d'affrontement et logique de maintien des populations sur le territoire local Les différentiations entre les pratiques migratoires relatives à la gestion de l'insécurité alimentaire sont de plusieurs ordres. Elles tiennent compte à la fois du temps et de l'espace. Elles tiennent compte également de la main-d'œuvre familiale disponible et de l'état de la production agricole.

Les migrations saisonnières

La suspension des travaux des champs favorise d'importants flux migratoires saisonniers d'une partie de la force de travail et plus spécialement celle des jeunes paysans. Ces mouvements sont nécessaires quand on sait que souvent les greniers ne sont pas pleins et que de surcroît il faut payer l'impôt, l'habillement des membres de la famille etc. Les migrations saisonnières consistent en l'absence de leurs acteurs pendant la totalité ou partie de la saison sèche ou saison des migrations. La migration 
est la principale activité pratiquée pendant cette période au regard de l'importance du nombre de personnes mobilisées. Son intérêt réside dans le fait qu'à part les revenus qu'elle procure aux familles, elle permet aussi aux acteurs de participer aux travaux agricoles. Selon ce schéma classique, les acteurs partent à la fin des travaux agricoles pour ne revenir qu'avant ou pendant ceux de la campagne agricole suivante. L'ensemble des migrants de retour à Taroum et Mogonana ont déclaré avoir migré à la fin des travaux agricoles. Les départs et les retours sont échelonnés dans le temps de façon à ne pas perturber l'équilibre économique déjà précaire de certaines unités de production. Cependant, cette stratégie peut perdre de son efficacité si par exemple la production agricole est très mauvaise, ce qui est le cas ces dernières années dans la région. Le retour des migrants pendant la saison des pluies s'accompagne souvent d'apport de nourriture au moment où les greniers sont vides et où la soudure s'est déjà installée. De plus en plus, les mauvais résultats de l'agriculture amènent certains migrants à prolonger leur séjour.

Les migrations annuelles

Elles prennent de plus en plus d'importance en raison non seulement de l'incapacité des acteurs à atteindre les objectifs fixés dans le cadre de leurs projets migratoires, mais aussi de la persistance des mauvais résultats de l'agriculture. Il est plus intéressant de chercher de l'argent pour payer des vivres pendant la période des récoltes où les prix sont à leur bas niveau que de participer à des travaux agricoles dont les résultats seront de toute façon décevants. Ce type de migration a l'inconvénient de réduire le temps passer au village de départ et concerne surtout les migrants séjournant dans les pays étrangers. Le plus souvent certains chefs de famille encouragent la prolongation des séjours notamment si la main-d'œuvre disponible peut faire face aux travaux agricoles.

Les migrations de courtes distances

41 L'expression, "migrations de courtes distances", est relative d'autant plus que les moyens de communication ont rapproché les lieux (Decoudras et Landrieu, 1998:3). Cependant, pour les populations des villages de Taroum et Mogonana, la migration vers la Côte est considérée comme lointaine, alors que la proximité de Niamey permet d'envoyer rapidement des vivres au village quelle que soit la quantité. Cette stratégie est surtout l'œuvre des chameliers, des vendeuses de sable fin etc. La migration vers Niamey permet d'avoir régulièrement des nouvelles de la famille et de pouvoir effectuer de brefs séjours au village à l'occasion des événements familiaux. Dans le Zarmaganda en général, les pratiques migratoires de survie se font essentiellement vers Niamey.

La gestion de la main-d'œuvre familiale

Les rapports entre insécurité alimentaire et phénomène migratoire posent la question de la gestion de la main-d'œuvre au sein des familles. Cette problématique renvoie au vieux débat sur l'importance d'une progéniture nombreuse. Il ne s'agit pas de soutenir ici les thèses pro-natalistes, mais plutôt de montrer l'efficacité de la migration dans les stratégies économiques dans un contexte d'insécurité alimentaire lorsque la maind'œuvre au sein des familles est importante. La gestion de l'insécurité alimentaire se traduit par une organisation ingénieuse du travail: une partie des hommes part en migration alors que l'autre partie assure les travaux agricoles. Les migrants ont pour mission de fournir des ressources à la famille. Cette façon de composer avec l'extérieur peut se faire de façon tournante, c'est-à-dire que les membres de l'unité de production 
se relaient dans la pratique migratoire. C'est la stratégie mise en œuvre par une famille à Taroum qui compte sept bras valides. Chaque année, trois d'entre eux migrent en Côte d'Ivoire, alors que les autres assurent les travaux agricoles au village. La migration tournante permet donc à ces familles d'optimiser leurs revenus.

Lorsque la main-d'œuvre est insuffisante, ce sont le plus souvent les femmes et les enfants qui assurent les travaux agricoles. Avec les absences prolongées des migrants et si la compensation économique est faible, la production agricole s'en trouve affectée. Il en résulte une forte baisse de cette production, même en années de pluviométrie normale. Certaines familles rentrent dans un véritable cercle vicieux qui les empêche de s'adapter à l'insécurité alimentaire. De plus en plus vulnérables ces familles sont les plus disposées à une migration définitive.

Migration et production agricole : de la complémentarité à la marginalisation

L'analyse des interactions migrations/insécurité alimentaire soulève la question de l'évolution des systèmes de production. En effet, les revenus migratoires et les ressources agricoles locales s'inscrivent dans le cadre de stratégies économiques qui accordent une large place à l'activité migratoire en tant que source de revenus. Dans un contexte d'insécurité alimentaire permanente et d'extrême pauvreté, ce rôle de complémentarité dévolu aux revenus migratoires, ne peut que se renforcer davantage au point de devenir une stratégie de survie. C'est ce qui explique sans doute l'intensification de la circulation migratoire à Taroum et Mogonana où chaque année, plus de $70 \%$ des bras valides sont mobilisés par les migrations saisonnières, annuelles et pluriannuelles.

Lorsque la production agricole est nulle comme ce fut le cas pendant les années de paroxysmes climatiques, le rôle assigné à la migration peut se révéler important en allant jusqu'à marginaliser l'activité agricole. Dans ce cas, il y a substitution momentanée de l'activité migratoire à l'activité agricole : la première ravit la place de la seconde dans les systèmes de reproduction économiques des familles.

Malgré la relative flexibilité des stratégies économiques familiales qui tiennent compte $\mathrm{du}$ contexte agro-climatique et encaissent mieux les crises de subsistance, cette souplesse peut très vite se rigidifier si les ressources migratoires ne parviennent pas à couvrir les besoins des familles. On assiste alors à l'abandon du territoire local qui ne permet plus d'assurer, dans un contexte de crises de subsistance et par le biais de la migration, leur reproduction économique et sociale.

En fonction des années et de l'état des récoltes, les rapports entre ressources migratoires et production agricole peuvent varier de la complémentarité à la marginalisation. La variation de ces rapports est fortement tributaire de l'état des récoltes annuelles, lui-même dépendant essentiellement des aléas climatiques.

La migration est ainsi devenue une stratégie de gestion de l'aléatoire. La persistance de l'insécurité alimentaire a fini par ancrer la pratique migratoire dans les comportements des populations de cette région. L'organisation de la migration en fonction du calendrier agricole et de la force de travail entre bien dans cette logique. Les départs et les retours sont échelonnés dans le temps de façon à ne pas perturber l'équilibre économique des unités de production.

La gestion stratégique des réserves vivrières familiales

49 Dans le Zarmaganda, l'absence d'un membre de la famille, durant la saison sèche (d'octobre à mai en gros), constitue une bouche en moins à nourrir. En raison de 
l'importance relative des migrants, l'impact sur la consommation vivrière peut devenir significatif notamment quand il concerne plus de trois personnes. En deçà de ce chiffre, les quantités de nourriture consommées varient peu, au-delà par contre on constate un effet réel sur la consommation du ménage. Dans ce cas, la migration repousse l'échéance redoutable de l'épuisement des réserves disponibles. La migration soulage donc l'unité familiale d'une partie de ces charges. Cette gestion stratégique des réserves familiales est bien comprise des paysans du Zarmaganda qui encouragent beaucoup de jeunes à prendre les chemins de la migration d'autant qu'elle apporte toujours un plus à la famille. Ainsi certaines familles du Zarmaganda ont pris l'habitude de travailler à Niamey pendant toute la durée de la saison sèche. On conserve le mil récolté jusqu'au retour de la famille au village à la saison des pluies; ceci permettant de vaquer en toute sécurité alimentaire aux travaux agricoles.

50 On peut considérer, à la lecture de ce qui précède, que la migration d'un membre de la famille s'inscrit dans un projet de mobilité spatiale qui répond le plus souvent à des stratégies collectives. Derrière la recherche effrénée de ressources indispensables à la survie des familles se déploie toute une gamme de stratégies, les unes plus efficaces que les autres, qui sont cependant toutes orientées vers le maintien des acteurs, et de ceux qui en dépendent, sur un territoire local, lequel n'arrive plus à générer des ressources suffisantes pour permettre d'assurer leur subsistance.

Stratégie de contournement et logique d'abandon du territoire local

51 La migration cesse d'être une stratégie d'adaptation quand il y a abandon du territoire. La logique de contournement est la solution ultime, le dernier recours lorsque toutes les stratégies, quels que soient les espaces dans lesquels elles se déploient, ne permettent plus aux acteurs d'être en mesure d'assurer leur survie. En général, ce sont les familles les plus vulnérables qui migrent, celles qui ne peuvent pas résister à la crise en restant au village.

Des villages en déprise

La migration intervient de deux façons sur la dynamique démographique des deux villages enquêtés. Les départs, lorsqu'ils sont saisonniers, s'accompagnent d'une modification momentanée des structures démographiques. On remarque dans les villages une proportion importante de femmes et d'enfants pendant la saison sèche. En dehors de quelques jeunes dans la force de l'âge qui ont choisi de s'adapter à la situation par la vente du bois de feu, la quasi-totalité des bras valides emprunte chaque année les chemins de la migration.

53 Les épisodes de sécheresses enregistrées au cours des années 1980 furent à l'origine de la migration définitive de nombreuses familles à Niamey et dans d'autres régions rurales du Niger. Elles ont laissé derrière elles des cases qui se délabrent. Les départs représentent une saignée démographique importante qui peut s'évaluer en fonction de l'évolution des effectifs de population. Entre 1982 et 1988, la population de Taroum a connu une régression significative, passant de 2025 à 1701 habitants soit une diminution de $16 \%$. Entre ces deux dates 64 familles sont parties définitivement, à la suite de la disette dazaï en 1984. Ce délestage démographique a été observé dans presque tous les villages du canton de Tondikiwindi et dans une moindre mesure dans ceux de Ouallam et Simiri. Cependant, à l'échelle du Zarmaganda, les données chiffrées ne signalent pas de diminution exceptionnelle. On constate même que la population de la région a augmenté, passant de 178494 habitants en 1981-1982 à 190765 habitants en 1988 soit une croissance de $7 \%$. Notre connaissance du terrain nous permet d'expliquer 
ce constat par le fait que les migrants installés ailleurs n'ont pas été rayés des listes de recensement et ont tout au plus été considérés comme absents.

La déprise humaine au sein des villages a évidemment des effets importants sur l'espace cultivé et sur l'environnement. De nombreux champs ont été abandonnés par leurs propriétaires qui restent inexploités.

L'ancrage en ville

55 La ville de Niamey est depuis la période coloniale une destination pour les migrants de la région. Pendant les premières phases de son développement, Niamey a connu une forte expansion démographique. Entre 1932 et 1940, l'accroissement annuel était de $17 \%$ dont $15 \%$ d'apport migratoire, entre 1950 et $1960^{3}$, il était de $12 \%$ dont $10 \%$ d'apport migratoire (Motcho, 1991: 52). Après l'indépendance, les stratégies et les modèles de développement des années 1970 avaient été essentiellement axés sur les investissements dans l'industrie et sur la modernisation de l'État. Cela s'était essentiellement traduit par l'installation de structure administrative et l'équipement des centres urbains renforçant ainsi la migration en provenance du monde rural (Kalasa, 1996 : 408).

Les différentes sécheresses et famines qu'a connues l'Ouest du Niger ont également contribué à l'accroissement démographique de la ville. En 1931 le flux des victimes de la famine atteignait 3000 personnes pour une population de 2183 habitants (Motcho, 1991 : 51) soit une augmentation de $138 \%$. À la suite de la famine de 1973-1974, la population de Niamey a augmenté de 100000 habitants (Gado, 1998: 70). On estime qu'en 1974, 20000 victimes de la sécheresse dont 13000 Nigériens parmi lesquels de nombreux originaires du Zarmaganda et du Tondikandia, gravitaient autour de Niamey dans les huttes en tige de mil en attente de l'aide alimentaire et exerçant des petits métiers de survie (Sidikou, 1975 : 208). En 1984-1985, au lendemain de la famine Dazaï, la population de Niamey a augmenté d'un tiers (Gado, 1998 : 70).

Niamey accueille aussi pendant la saison sèche beaucoup de migrants qui constituent une véritable population «flottante ». On dénombre des originaires du Zarmaganda à la recherche des moyens pour assurer la subsistance de la famille restée au village. Pour nombre d'entre eux, la proximité de Niamey permet d'aider rapidement la famille. Dès que le migrant gagne un peu d'argent, il peut acheter de la nourriture et l'expédier au village. Cette pratique est surtout celle des chameliers spécialisés dans l'approvisionnement de la ville de Niamey en bois de chauffe, en paille, en secco et en résidus agricoles (fourrage). Leurs séjours dans la capitale, qui s'organisent tout au long de l'année, visent à pallier les disettes, les famines et les soudures en achetant des vivres grâce aux revenus de leur vente.

L'ancrage en ville se traduit aussi par le développement d'un habitat de type spontané. Ces îlots que l'on appelle les Charégazamna («balayer pour s'installer » en Haoussa) ou "ronds-points", sont construits en paillote. Ils forment à côté des habitats modernes de véritables enclaves tout à fait remarquables dans les paysages urbains. Ils sont le plus souvent occupés par les originaires du Zarmaganda, du Tondikandia et d'autres ethnies (Haoussa, Bella, Gourmantché, Touareg etc.). Ces populations vivent constamment sous la menace d'incendies et de destruction, subissant la "politique du bulldozer ». Quand les Charégazamna sont détruits, les victimes vont s'installer sur d'autres sites. L'une des caractéristiques de ces îlots est leur grande mobilité. Leur déplacement s'opère en fonction de la mise en valeur du site sur lequel ils sont construits. La plupart de ces installations sont construites dans des espaces vides à la 
périphérie de certains quartiers (Motcho, 1991 : 78). On les retrouve également en plein cœur de certains quartiers où les migrants n'hésitent pas à investir les terrains vagues, les espaces réservés à la construction d'une structure sociale, les rues etc.

Les migrations qui aboutissent à la création de ce type d'habitat sont généralement définitives et les retours au village sont très rares. En outre Niamey est devenue un lieu de repli momentané et parfois durable pour les populations victimes de crises. Suite à la famine de 1984, elle a constitué un refuge pour 20 familles de Taroum et 12 de Mogonana. En 1984, Mogonana a été quasiment abandonné au profit de Niamey pendant toute la saison sèche. La plupart de ces familles se sont éparpillées dans les îlots d'habitat spontané et les quartiers périphériques notamment Koiratégui où existe un secteur dénommé Taroum.

En 1995 les migrations en direction des villes concernent $71 \%$ des migrants de Mogonana et $53 \%$ de ceux de Taroum. La ville est perçue, à juste titre, comme un lieu non tributaire des conditions climatiques. Niamey joue donc une fonction d'accueil des réfugiés de la sécheresse (Bruneau et al., 1995 : 249).

61 Les migrants installés ou en séjour dans les villes pratiquent pendant une bonne partie de l'année des activités qui relèvent essentiellement du secteur informel. En côte d'Ivoire et au Bénin, ils sont colporteurs. À Niamey, la gamme de leurs activités est variée et relève de la débrouille.

L'image des gens du Zarmaganda qui s'est construite dans la capitale est celle de chameliers errants en toute saison dans les rues à la recherche d'une hypothétique clientèle urbaine, de Takalakoy ${ }^{4}$ vendeuses de copto ou de sable fin n'hésitant pas à recourir parfois à la mendicité pour calmer les douleurs d'un ventre vide. Cette image a fini par conforter les représentations dévalorisantes et même méprisantes que certains citadins ont de ces migrants considérés comme des affamés se réfugiant dans la ville à la fin de la saison des pluies.

La fixation des Zarma dans les autres régions rurales

Les migrations liées à l'insécurité alimentaire se traduisent par un remaniement des structures d'occupation de l'espace dans les aires de colonisation agricole. De nouvelles dynamiques spatiales se créent transformant les paysages. Mais avant d'aborder la recomposition des espaces liée à la fixation des Zarma, il convient d'envisager la manière dont les migrations agricoles liées aux crises alimentaires ont évolué (cf. carte $\left.\mathrm{n}^{\circ} 2\right)$.

64 La recherche de nouvelles terres de culture a toujours été une motivation importante pour ces populations. Ces mouvements constituent une des dimensions importantes de la dynamique de l'occupation de l'espace. Il faut savoir que le peuplement du Zarmaganda répond en partie à la logique de recherche de nouvelles terres parfois au gré de la chasse $\mathrm{e}^{5}$ ou suite à l'épuisement des sols cultivés. Ces déplacements que l'insécurité pré-coloniale rendait dangereux s'étaient développés pendant la période coloniale. Les famines ont également joué un rôle dans les implantations agricoles à caractère définitif dans certaines régions notamment la vallée du fleuve Niger (Sidikou, 1974). Cependant, ces processus diffèrent sensiblement des mouvements de colonisation observés ces vingt dernières années. Ils sont de véritables mouvements à grandes distances.

65 Les sécheresses des années 1980 constituent le point culminant de ces migrations dans le Zarmaganda. Elles sont également le résultat d'une prise de conscience des migrants 
de la gravité de la crise alimentaire et de son irréversibilité dans les villages de départ. Elles révèlent aussi l'incapacité des migrations saisonnières ou de longue durée vers Niamey et les pays côtiers à répondre aux situations de grave pénurie. Même si leurs destinations sont éclatées, deux régions absorbent l'essentiel de ces mouvements. Il s'agit essentiellement de Say et Kollo.

Ces régions possèdent des conditions agro-écologiques favorables notamment à Say. La position méridionale de cette région (située dans l'extrême sud-ouest du pays) fait qu'il existe un climat de type soudano-sahélien. Les précipitations annuelles sont de 575,6 mm (période 1961-1990) avec un coefficient de variation de $23 \%$ (Amadou, 1994). Par ailleurs, les densités d'occupation humaine sont faibles dans certains cantons et favorisent par conséquent l'implantation d'immigrants. En plus, l'existence de terres vierges soustraites à l'onchocercose dans les années 1970 ne pouvait que favoriser leur fixation ${ }^{6}$.

Les mouvements de colonisation dans les départements de Say et Kollo remontent au début des années 1970 à la suite de la sécheresse de 1973-74. Ils étaient de très faible ampleur et les retours aux villages d'origine étaient fréquents. Les migrations vont se développer surtout au cours des années 1980. En 1982 le chef de l'État, Seyni Kountché, dans son message du 15 Avril, invita les habitants des centres urbains (fonctionnaires employés, militaires, commerçants élèves et étudiants) à participer davantage au développement de la production vivrière nationale ${ }^{7}$. Cet appel a été précédé du déclassement d'une partie de la réserve de Tamou (qui couvre $140000 \mathrm{Km}^{2}$ ). Cette zone a été redistribuée aux gros exploitants (fonctionnaires et commerçants) de Niamey. L'opération, dénommée Aï noma ( «ultivons»), a permis aux exploitants d'employer une main-d'œuvre en provenance notamment du Zarmaganda. Le contact des premiers migrants avec les autochtones peuls qui leur ont prêté des terres, va entraîner progressivement leur installation durable dans la région où ils seront rejoints par les Songhaï et les Bella de Téra, les Kourfey du département de Filingué. Les villages de Taroum et Mogonana ont aussi alimenté ces migrations.

68 Les immigrants s'installent de préférence en dehors des villages d'accueil, dans des hameaux de culture homogène du point de vue ethnique et que l'on dénomme Zarmagandey. Les originaires du Zarmaganda pratiquent l'agriculture vivrière en reproduisant les systèmes de production tels qu'ils les pratiquaient dans leurs zones de départ.

69 L'importance du volume de ces migrations de colonisation agricole peut être appréhendée de façon approximative par une analyse des résultats du Recensement Général de la Population. Les départements de Say et de Kollo avaient en 1988 les taux annuels d'accroissement les plus élevés des régions du fleuve et du Dallol-Tapoa : 4,8\% et $5,3 \%$ contre $2,7 \%$ pour Ouallam et $3,2 \%$ pour l'ensemble du pays 9 .

70 La densité de population est passée de 13,9 habitants $/ \mathrm{km}^{2}$ en 1977 à 24,6 habitants $/ \mathrm{km}^{2}$ en 1988 dans le département de Kollo, et de 7 habitants $/ \mathrm{km}^{2}$ à 11,6 habitants $/ \mathrm{km}^{2}$ dans celui de Say, alors que pour la même période on observe une augmentation très timide de ces densités dans le département de Ouallam (6,5 habitants $/ \mathrm{km}^{2}$ en 1977 et 8,6 habitants $/ \mathrm{km}^{2}$ en 1988).

71 Il est cependant difficile de déterminer la part de l'immigration dans cette dynamique démographique comme facteur externe. Le nombre de personnes originaires du Zarmaganda qui se seraient définitivement installées dans les départements de Téra, 
Tillabéri, Kollo et surtout Say était estimé en 1987 de 12000 à 15000 soit environ $9 \%$ de la population totale du département (Sidikou, 1991).

La colonisation des terres s'est traduite par la création de nombreux hameaux et villages spatialement dispersés, dont certains, en raison de leur importance démographique et $\mathrm{du}$ souhait de leurs habitants, ont été érigés en villages administratifs avec des chefs de villages attitrés. Dans le département de Say, il s'agit de Séno Konkédjé dit Kokaïna, dans le canton de Tamou, officiellement créé en $1988^{10}$, de Sabarabangou (74 familles, 691 habitants en 1998), Goulbel (53 familles, 577 habitants en 1998) dans le canton de Say ${ }^{11}$. Dans le département de Kollo, deux hameaux de culture, Banizoumbou et Zarmagandey dans le canton de Kirtachi, ont fait l'objet d'une demande de création de village administratif en examen au Ministère de tutelle. Dans celui de Karma, un autre hameau peuplé d'originaires du Zarmaganda a entrepris des démarches similaires.

La fixation de ces populations soulève la question de leur dynamique territoriale. Dans le Zarmagandey de Koikoirey-zarma créé en 1984 par des originaires de cinq villages du Zarmaganda, les immigrants étaient unanimes sur le caractère temporaire de leur établissement. Ils estiment que les terres qui leur ont été prêtées par les autochtones ne produisent pas assez pour les nourrir. Beaucoup d'entre eux envisagent de continuer leur descente méridienne. La dynamique territoriale de ces immigrants ne s'arrête donc pas à ce hameau de colonisation.

Un espace migratoire en recomposition et en extension permanentes

Les pratiques migratoires liées à l'insécurité alimentaire permettent également de comprendre l'évolution de l'espace balayé par les flux des populations de Taroum et Mogonana. Depuis le début des migrations modernes, l'espace migratoire des populations de ces deux villages n'a cessé de s'étendre et de se recomposer. À une migration internationale, orientée vers la colonie de Gold Coast, a succédé des flux réguliers et soutenus vers le Nigeria pendant les années 1970. Aujourd'hui, les départs vers l'étranger sont essentiellement polarisés sur la Côte d'Ivoire qui a accueilli $11 \%$ des absents de Taroum en 1995 et 33 \% de ceux de Mogonana pendant la même année. Ils concernent dans une moindre mesure le Bénin (six familles de Taroum), le Togo (un ressortissant de Mogonana) et l'Arabie Saoudite (deux ressortissants de Taroum y séjournent en 1995). L'architecture spatiale des flux révèle une multiplication des lieux d'accueil et d'implantation des migrants originaires de nos deux villages. La migration définitive de plusieurs familles dans le département de Say consacra un nouveau tournant dans la dynamique de l'espace migratoire. Aux migrations rurales-urbaines sont donc venues s'ajouter des migrations rurales-rurales.

L'espace migratoire se recompose au gré de la création de nouveaux courants et de l'essoufflement des anciens. Cette dynamique spatiale des migrations s'inscrit dans le cadre d'échelles emboîtées. Les territorialités des populations sont plurielles. À part le territoire de départ qui est un espace de vie pour certains et un territoire de référence pour les autres, ceux qui se sont établis ailleurs, les gens de Taroum et Mogonana, s'approprient d'autres lieux souvent éloignés de leurs villages d'origine; dans la plupart des cas la ville. Les dimensions de l'espace ressource sont à la fois nationales, régionales et internationales.

Conclusion

76 Nous avons pu apprécier le rôle joué par la migration dans la gestion de l'insécurité alimentaire. Les pratiques migratoires répondent à deux logiques principales. La 
première consiste à avoir recours à la migration dans le but de maintenir l'unité familiale dans le territoire local. En cherchant constamment à l'extérieur du territoire local les solutions à la survie, les populations sont dépendantes des ressources migratoires; ce processus explique sans doute l'intensification de la circulation dans la région. Une autre stratégie vise à préserver la production agricole familiale en organisant le départ de certains membres de la famille. Il soulage momentanément l'unité familiale du point de vue de l'autoconsommation vivrière.

77 La deuxième logique consiste à adopter la migration pour abandonner le territoire local. Cette attitude s'observe de façon épisodique lorsque les crises de subsistance sont graves. Il s'agit de transférer des hommes et leurs activités vers de nouveaux territoires. Cette forme de migration est exceptionnelle à Taroum et Mogonana. Une des conséquences est la régression démographique lorsqu'elle prend de l'ampleur.

Ces deux logiques traduisent chacune une attitude face aux crises de subsistance. La première consiste à affronter l'insuffisance alimentaire en cherchant à s'y adapter, tandis que la seconde vise à la contourner en abandonnant les territoires qui sont menacés. Par ailleurs, la migration peut contribuer au renforcement de l'insuffisance alimentaire, notamment, lorsque l'absence des bras valides n'est pas compensée par une main-d'œuvre de substitution, et surtout par des ressources migratoires.

79 L'incertitude de la production agricole, à laquelle viennent s'ajouter les difficultés d'accès aux autres sources de revenus locales, témoigne de la nécessité de recourir à des ressources extérieures au territoire local. C'est là que les diverses pratiques migratoires, révélatrices chacune de stratégie familiale spécifique, prennent toute leur signification. Les unes s'inscrivent dans une logique de diversification des sources de revenus et de stratégie de survie, les autres tendent vers l'effritement des structures familiales villageoises par la migration définitive de certains de leurs membres. Dans le premier cas, il y a, en fonction des vicissitudes de la production agricole une dépendance accrue à l'égard des ressources migratoires. Dans le second, la migration sert de passerelle pour un nouvel ancrage, en ville ou dans d'autres milieux ruraux en particulier, à travers la création de nouveaux territoires. On voit déjà à Taroum, et au plus fort des crises de subsistance, une régression des effectifs de la population villageoise qui ne doit cependant pas être assimilée aux déséquilibres démographiques momentanés, qui résultent des migrations saisonnières, observés dans presque tous les villages du canton de Tondikiwindi et du département de Ouallam.

80 La question cruciale se pose donc du devenir de ces sociétés et de la viabilité de leurs territoires. Il est vrai que la plupart des villages du canton de Tondikiwindi sont en marge de la limite nord des cultures et que cette position géographique explique en partie le caractère aléatoire de la production agricole. Il est vrai aussi que les ressources migratoires ont permis jusque-là aux populations d'atténuer les effets de l'insécurité alimentaire. Mais, cette souplesse, ce réajustement continu et précaire peut-il être durable? Certes, les sociétés locales ont toujours trouvé des solutions aux déséquilibres de leur milieu, mais les pratiques migratoires ne peuvent constituer des solutions sur le long terme même si la migration est une pratique spatiale séculaire. Dans un contexte d'insuffisance alimentaire chronique, le rôle de la migration dans le maintien des populations sur leur territoire local risque de perdre de son efficacité. Il est alors fort probable que la migration crée progressivement le vide dans ces milieux.

81 Là où les pouvoirs publics préoccupés par l'aménagement du territoire, tentent avec plus ou moins de succès, et à travers les projets de développement - certains 
comportant des volets food for work et cash for work - de maintenir en l'état la distribution des hommes, il convient de s'interroger sur la portée réelle de ces actions. Peuvent-elles maintenir des populations qui ont intégré la pratique migratoire dans leur système de production? Ne faut-il pas aider à la promotion d'autres activités génératrices de revenus et capables de fournir une alternative crédible aux migrations définitives? Ne faut-il pas considérer la migration comme n'importe quelle activité et chercher à mieux la rentabiliser?

\section{BIBLIOGRAPHIE}

AMADOU Boureïma (1994) Crise alimentaire et conquête agricole des nouvelles terres dans le Sud-ouest du Niger, Revue de Géographie Alpine, vol. 2, pp. 171-184.

AZOULAY Gérard et DILLON Jean-Claude (1993) La sécurité alimentaire en Afrique. Manuel d'analyse et d'élaboration de stratégies, Paris: ACCT-Karthala, 269 p.

BRUNEAU Jean-Claude (1994) et al. Villes nigériennes, l'émergence d'une armature urbaine nationale en pays sahélien, Revue de Géographie Alpine, vol. 2, Numéro hors série, pp. 241-258.

DECOUDRAS Pierre-Marie et LANDRIEU Sophie (1998) Les réseaux au secours du territoire local. Dynamique contemporaine des Touaregs de la vallée de Tidène (massif de l'Aïr, Niger) In Les montagnes tropicales : identités, mutations, développement. Talence : DYMSET ; CRET, Table-Ronde, 27 et 28 novembre $1998,17 \mathrm{p}$. à paraître.

DERRIENIC Hervé (1977) Famines et domination en Afrique. Paysans et éleveurs sous le joug. Université de Haute Bretagne, Rennes, $343 \mathrm{p}$.

DUBRESSON Alain et al. (1994) Les Afriques au sud du Sahara, Belin/Reclus, Montpellier, 480 p.

EBRAHIMI-YEGANEH Fatimeh (1996) Les problèmes alimentaires dans la région soudano-sahélienne de l'Afrique occidentale (le cas du Burkina Faso, du Mali, du Niger et du Sénégal), Université de Bordeaux III, Bordeaux, $407 \mathrm{p}$.

GADO Boureïma Alpha (1998) Environnement et pauvreté au Niger : crises écologiques, migrations et pauvreté urbaine, Annales de l'Université Abdou Moumouni de Niamey, numéro hors série, pp. 63-77.

GADO Boureïma Alpha (1992) Concepts traditionnels sur les sécheresses, famines et épidémies du Sahel, Sécheresse, 3 (4), pp. 243-250.

GADO Boubé (1980) Contribution à l'histoire des populations d'entre Niger et Dallol Mauri, Etudes Nigériennes, $\mathrm{n}^{\circ} 45,350 \mathrm{p}$.

HAMA Boubou (1967) Histoire traditionnelle d'un peuple : les Zarma-Songhaï, Présence Africaine, Paris, $278 \mathrm{p}$.

JOUVE Philippe (1991) Sécheresse au Sahel et stratégies paysannes, Sécheresse, 2 (1), pp. 61-69.

IDRISSA Kimba (1981) Guerres et sociétés. Les populations du « Niger » occidental au XIXe siècle et leurs réactions face à la colonisation (1896-1906), Etudes Nigériennes, n 46, 222 p. 
KALASA Benoît (1996) Crise et perspectives d'urbanisation dans les pays du Sahel In Jean Coussy et Jacques Vallin, Éds. Crises et population en Afrique, CEPED, Paris. pp. 407-422.

LAVIGNE DELVILLE Philippe (1996) Gérer la fertilité des terres dans les pays du Sahel. Diagnostic et conseils aux paysans, CTA, Saint-Etienne, $397 \mathrm{p}$.

LUXEREAU Anne et ROUSSEL Bernard (1997) Changements écologiques et sociaux au Niger. Des interactions étroites, L'Harmattan, Paris, 238 p.

MAUNY Raymond (1961) Tableau géographique de l'ouest-africain au Moyen Âge d'après les sources écrites, la tradition orale et l'archéologie, IFAN, Dakar, 587 p.

MOTCHO Henri Kokou (1991) Cadre de vie et système de santé à Niamey, Université de Bordeaux III, Bordeaux, $310 \mathrm{p}$.

MOUNKAILA Harouna (1999) Migrations, Environnement et Santé au Niger. Dynamique récente des migrations Songhaï-Zarma dans le département de Tillabéri (Ouest du Niger), Université de Bordeaux III, Bordeaux, $433 \mathrm{p}$.

MOUNKAILA Harouna (1997) Conditions du milieu et dynamique migratoire dans le Zarmaganda, Projet Niger 20, Université Abdou Moumouni de Niamey, Niamey, 33 p.

MOUNKAILA Harouna (1994) Analyse dynamique et étude typologique de la mobilité au Sahel ouestafricain, Université de Bordeaux III, Bordeaux, $120 \mathrm{p}$.

MOUNKAILA Harouna (1993) Mobilité et mode de vie dans une région sahélienne en crise: l'exemple du canton de Simiri dans le Zarmaganda, Université Abdou Moumouni de Niamey, Niamey, 158 p.

OLIVIER DE SARDAN Jean-Pierre (1984) Les sociétés Songhaï-Zarma (Niger-mali). Chefs, Guerriers, esclaves, paysans..., Karthala, Paris, 299 p.

OUSSEINI Issa (1996) Les sécheresses au Niger : clichés et réalités In RESADEP-Institut PANOS Environnement au Niger, NIN, Niamey, pp.16-23.

REPUBLIQUE DU NIGER. MINISTERE DU PLAN, Étude monographique de l'arrondissement de Ouallam, Niamey, 1999, 45p.

ROBIN Nelly (1996) Atlas des migrations ouest-africaines vers l'Europe 1985-1993, ORSTOM, Paris, 109 p. SIDIKOU Arouna Hamidou (1991) Une région sahélienne en crise : le Zarmaganda (République du Niger) In Jean Gallais et al. (Dir.), Sahel, Nordeste, Amazonie. Politiques d'aménagement en milieux fragiles, Paris, UNESCO, L'Harmattan, pp. 51-86.

SIDIKOU Arouna Hamidou (1974) Sédentarité et mobilité entre Niger et Zgaret, Études Nigériennes, $\mathrm{n}^{\circ} 34,250 \mathrm{p}$.

TRAORE Sadio (1997) Conséquences des migrations sahéliennes, Pop Sahel, n²6, pp. 20-22.

TRAORE Sadio (1997) Migrations et urbanisation en Afrique de l'Ouest: résultats préliminaires, CERPOD, Bamako, $31 \mathrm{p}$.

TORNEZY Robert Rapport de tournée effectuée dans les cantons de Hamdallaye et Farka en 1934, Archives Nationales du Niger, Niamey, 1934, 17 p.

URVOY Yves (1936) Histoire des populations du Soudan central, Larose, Paris, $350 \mathrm{p}$.

ZACHARIAH Kunniparampil Curien (1978) et al. Aperçu sur les migrations en Afrique de l'Ouest, OCDE, Paris, $87 \mathrm{p}$. 


\section{NOTES}

1. - Chef de guerre.

2. -TORNEZY, Rapport de tournée effectuée dans les cantons de Hamdallaye et Fakara en 1934. Niamey : Archives Nationales du Niger, 1934, 17 p.

3. -La population est passée de 11790 habitants en 1950 à 29950 habitants en 1960.

4. -Femmes portant sur leurs épaules un équipement en forme de balancier constitué d'un bâton aux extrémités duquel sont suspendues des cordes qui servent au transport d'objet.

5. -Quand, au cours de la chasse, les individus découvraient des espaces propices à l'agriculture, ils s'y installaient avec leurs familles après les avoir défrichés.

6. -L'éradication de l'onchocercose fait suite au programme de l'OMS au début des années 1970.

7. -Le Ministre de la fonction publique a procédé au réaménagement des horaires de travail en libérant le samedi matin pour permettre les activités de la terre.

8. -Décret n 76 141/PCMS/MDR du 22 août 1976.

9. -Recensement général de la population (RGP) 1988.

10. -Par décision du 29 janvier 1988 du Ministère de l'intérieur.

11. -Recensement administratif d'avril 1996 effectué par le service d'État-civil de la sous-préfecture de Say.

\section{RÉSUMÉS}

Les profondes mutations, amplifiées par les sécheresses récentes que connaît le Zarmaganda (Niger) constituent une des clés d'interprétation de son dynamisme migratoire. En effet, elles se manifestent notamment par la persistance d'une insécurité alimentaire qui contribue largement à l'accélération des migrations, perpétuant ainsi le développement de cette pratique spatiale séculaire. Cet article met en évidence les interactions complexes entre insécurité alimentaire et migration. Dans le Zarmaganda, la gestion de l'insécurité alimentaire s'exprime à travers des pratiques migratoires diverses qui s'inscrivent soit dans une logique de maintien des populations dans leur territoire, soit dans la finalité de les soustraire de cette contrainte majeure. Il en résulte une inscription spatiale différenciée de ces migrations en fonction des logiques qui les soustendent.

From Circular Migration to the Abandonment of Local Territories in the Zarmaganda (Niger) -The deep transformations amplified by recent droughts which have hit the Zarmaganda are major keys in interpreting migratory phenomenon. These transformations mainly take the form of an enduring food insecurity which largely contributes to the acceleration of migrations, thereby perpetuating the development of this age-old spatial practice. This articles aims at showing evidence of the complex interactions between food insecurity and population movement. In the Zarmaganda, the management of food insecurity is reflected in various migratory practices. These practices are either consistent with the strategy of maintaining populations in their territory or aimed at removing them from this major constraint. This leads to different spatial consequences according to the processes which underlie them. 
De la migración circular al abandono del territorio local en el Zarmaganda (Níger). -- Las profundas mutaciones, amplificadas por las recientes sequías, constituyen una de las claves de interpretación del actual dinamismo migratorio que experimenta el Zarmaganda. Dichas mutaciones se manifiestan particularmente a través de una inseguridad alimenticia persistente. Ésta situación contribuye a acelerar las migraciones y, así, a perpetuar el desarrollo de esta secular práctica espacial. Este artículo muestra las interacciones complejas existentes entre la inseguridad alimenticia y la migración. En el Zarmaganda, la gestión de la inseguridad alimenticia da lugar a prácticas migratorias que obedecen a lógicas diversas. Éstas pueden perseguir el mantenimiento de las poblaciones en sus territorios, o al contrario tener como finalidad de sustraerlas de este riesgo. De esta diversidad resulta una inscripción diferenciada de las migraciones en el espacio, dependiendo del objetivo perseguido.

INDEX

Mots-clés : causes des migrations, territoire, environnement, famine

Index géographique : Niger

\section{AUTEUR}

\section{HAROUNA MOUNKAÏLA}

Géographe, Université Abdou Moumouni de Niamey (Niger), École Normale Supérieure, BP 10963 Niamey. 